\title{
A CORELESS APPROACHES FOR ON/OFF MARX TYPE MODULATORS
}

\author{
Anatoly Krasnykh, Stanford Linear Accelerator Center, Menlo Park, CA 94025
}

\begin{abstract}
SLAC was first to report using ON/OFF switches in Marx type modulator. The development of Marx type modulator was bounded with the NLC need. The high energy physics runs based on the ILC concept where longer modulator pulse width is required. The SLAC idea of coreless modulators was useful for other applications (medicine, military, home security, etc.). The discussed conception is presented as a continuation of the earlier published articles. Several types of the Marx ON/OFF type modulators are under consideration. This article describes the new coreless approach, based on the solid state ON/OFF Marx's topology. An AC high voltage network feeds individual Marx's cells through the inductive and diode assemblies. Further integration of the ON/OFF Marx type modulator and its power supply is proposed. Two topologies are under consideration. The first scheme is an integration of DC/DC converters with ON/OFF Marx. The second topology is based on the usage of AC network directly with ON/OFF Marx scheme.
\end{abstract}

\section{INTRODUCTION}

In the last five years, two general conceptions of the 3 usec pulse width modulator were studied. The first was an 8-pack induction type modulator with solid-state switches. The other is a so- called hybrid modulator where a solid-state assembly is proposed instead of the thyratron switch. Both concepts imply a ferromagnetic media for transformation of the power. The energy transfer efficiency through a conversion system is one important parameter, where the large number of modulators are used (for example, the former ILC case). As it has been shown, both general conceptions look promising for the requested 3 usec pulse width. Presently the problem of the energy transfer efficiency is not a sufficient problem, but may be become an important when the pulse duration is less than 2 usec. Although the problem of energy transfer efficiency for modulators with a short pulse was analyzed earlier in paper $^{2}$, the manifestation of this problem may be seen in the future. One attractive advantage of the Marx method is the high voltage is not present continuously and there is no ferromagnetic media. The high voltage is erected only for the pulse duration and decays back quickly to the primary supply voltage between pulses. The energy is delivered to the load during the discharge mode and is reimbursed during the time between pulses. The changing modulator specification, for example from 2 to 6 usec, will not effect the modulator redesign. A pulse width modulation or a pulse train is possible.

\section{MARX'S TOPOLOGIES AND EFFICIENCY}

There are two Marx topologies ${ }^{1}$, which are show in Fig.1A and Fig. 1B accordingly.

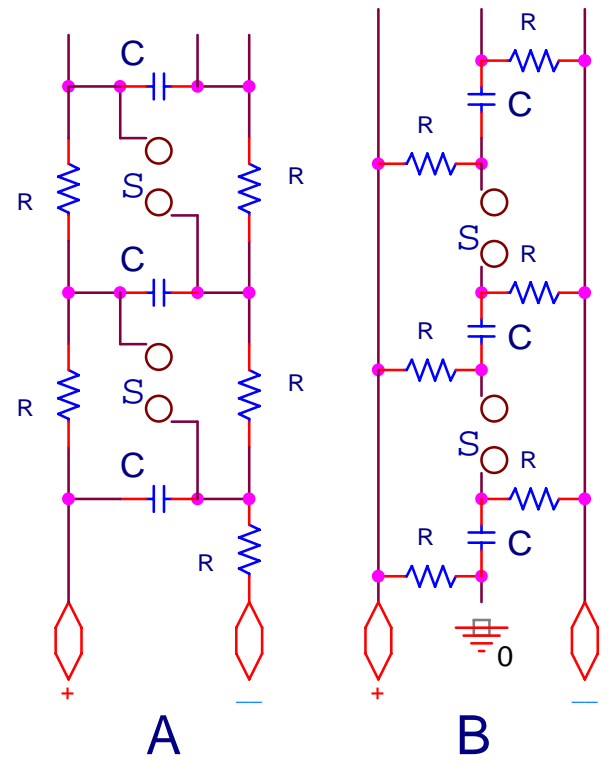

Fig. 1 Two (A and B) original Marx schemes $\mathrm{C}$ is storage energy capacitors, $\mathrm{R}$ is resistive ladder networks, and $\mathrm{S}$ is switches.

In well-known topology A, two arrays of resistive arms (or so-called ladder networks) are used. They are the recharging and isolation means. During the discharge mode, the arms shunt a load. The resistive ladder of each arm must be much higher than the load impedance. In that case, the accumulated energy of the capacitors is delivered to the load with a high efficiency. Only small amount energy is dissipated on the resistive ladders. However, the high value of charging resistors will hinder the efficiency and repetition rate of the Marx modulator. The resistive networks 
are not suitable for the modern solid state Marx modulators. The Marx circuitry is shown in article ${ }^{2}$ represents B topology. NiZn ferrite isolation transformers are used for the recharge and isolation modes. The other topology of the Marx pulser was also described earlier in the paper ${ }^{2}$ too. There, the active recharge switches were proposed.

The disadvantage of aforementioned topologies is either low efficiency of the resistive ladder networks or the complexity of the active switches involved.

The analysis of the Marx scheme shows that during the discharge mode, it is necessary to employ some kind of element in order to isolate the outputs of the power supply units and the Marx's cells. These elements must hold off the cell pulsed voltages. As it was noted above, the high resistive ladder network is not suitable for modern modulators due to low efficiency.

The high efficiency can be achieved if inductance ladder networks replace the resistor networks and the pulse duration $t_{p}$ is much smaller than the $L / R_{\text {load }}$ time constant, where $L$ is the total inductance of the charging arm and $R_{\text {load }}$ is the load impedance. During the time between pulses, the power supply effectively restores the charge in the storage capacitors. Due to the inductive arms, there is good isolation between the primary source and the Marx output during the discharge mode.

It was discovered that mutual coupling between the stage inductors could help in the efficiency of the Marx modulator. The improving of efficiency is due the fact that the direction of the flux in the coupling coils has an $180^{\circ}$ difference for discharge and recharge modes. Computer simulation shows that inductive elements are suitable to get the high efficiency. It seems that the inductive ladder networks will be rather expensive compared to the resistive networks. Other idea for the Marx modulator is to employ diode ladder networks. It has been found that the Marx topology A does not work if the diode ladder network is used in both arms. However, the modulator works if the diode networks are used in topology B. The proposed Marx modulator circuitry is presented in Fig. 2. Two Marx cells are shown to simplify the picture. The recharge and isolation ladder networks are used here in the B topology. The cell capacitors C are partially discharged during the pulse, and are recharged through two diode assemblies. The diode assemblies isolate the DC power supply during the discharge mode.

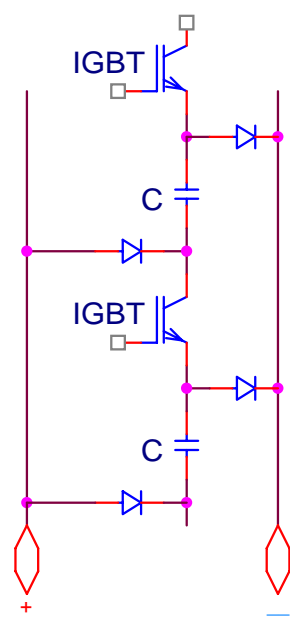

Fig. 2

The difference between proposed circuitry and the original Marx scheme is a fact that:

- The semiconductor switches are used to control the partial discharge mode and the pulse duration

- The passive semiconductors are employed in both arms.

Above Marx topology must have the special DC power supply.

\section{POWER SUPPLY FOR CORELESS TOPOLOGY}

Another question is what kind of charging power supply would be suitable for the proposed coreless topology? Before describing it, let us look at the traditional, widely used power supply topology. Of course, traditional power supplies can be directly employed for the proposed modulator. Traditionally, the power supply consists of AC to DC elements, a filter capacitor bank, a charging choke, a diode assembly (or a command charging unit), and a DeQing circuit to regulate the output voltage of the modulator. This topology is the present SLAC power supply scheme. Apparently, this topology is rather complicated and expensive. The employment of semiconductor elements in the ladder networks yields an advantage: to use AC power directly without any intermediate elements. Topology B, with the diode assemblies, is shown in Fig. 3. However, secondary winding in this topology must hold off whole pulse voltage. The left side of diode stack is in conductive mode and HV pulse applies for secondary winding. We think that the proposed topology may be attractive for the middle range of output voltages. For example, the range $30-150 \mathrm{kV}$ is reasonable for the high 
efficient isolation $\mathrm{x}$-fmrs. The novel approach of Marx type modulator topology greatly simplifies the charging circuitry, since there is no need for a filter capacitor bank, charging choke, command charging unit and DeQing elements.

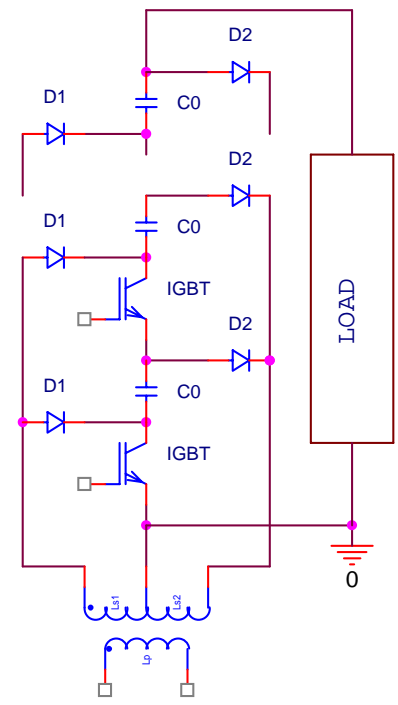

Fig. 3

The Marx type modulator consists of the capacitive load array that is an energy accumulator. No additional filter capacitor bank is necessary. The elimination of the filter capacitor bank and other traditional power supply elements also makes the proposed topology simpler, and less expensive.

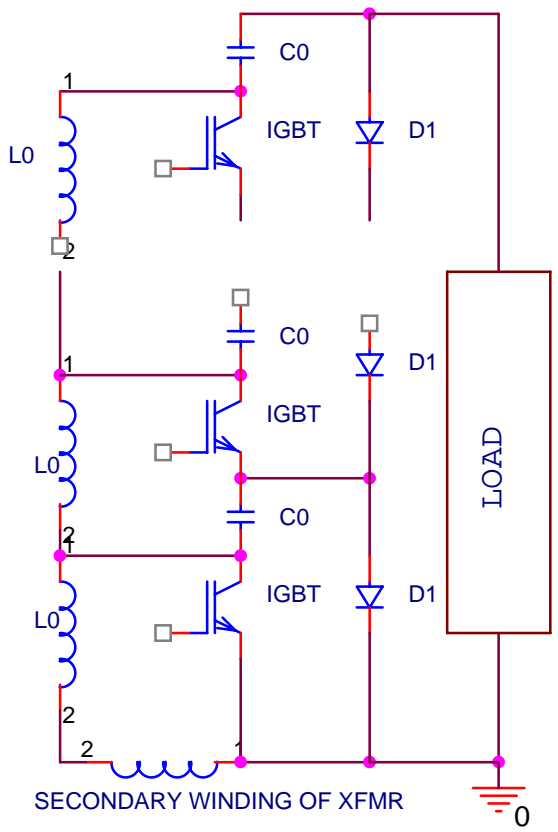

Fig. 4

Disadvantage of this topology is the need to have whole pulse isolation in the transformer. In final, the coreless approach of Marx topology with an AC power supply is shown in Fig.4. A three-phase version of circuitry needs more space to present. That is why it is shown one AC phase version. Primary circuitry is not shown. An inductive and diode ladder networks are used in topology $\mathrm{A}$ as an example. The IGBT current in three-phase version is small enough to employ middle range of IGBT package. This will benefit to the pulse rise time.

The AC integrated Marx coreless topology was experimentally tested to prove the principle. The experimental Marx circuit was simple and one $115 \mathrm{~V} 60 \mathrm{~Hz}$ AC phase was used. So, the diode assembly loss can apparently be smaller than stray capacitance loss. The next question is what would be the optimal primary wall plug AC voltage range for the coreless concept? In order to reduce the Joule loss, the primary AC voltage could be in the 2-10 kV range. Apparently, this level may no conflict with the maintenance and safety expenses. This question is still under study.

\section{CONCLUSION}

The possible conceptual designs of the Marx type modulator have presented. The efficiency of such a modulator is been higher if inductive and diode ladder networks are used as isolation and recharge means. The proposed modulator circuitry comprises the inductive and diode ladder networks. The analysis shows that coupled and uncoupled inductor topology may meet the efficiency requirements for Marx type modulators. Further integration of the ON/OFF Marx type modulator and its power supply is proposed. Two topologies are under consideration. The first scheme is an integration of DC/DC converters with ON/OFF Marx. The second topology is based on the usage of AC network directly with ON/OFF Marx.

\section{ACKNOWLEDGEMENTS}

Author would like also to thank everybody from Klystron and Microwave Department who was involved in the discussed subject.

This work supported by Department of Energy under contract DE-AC03-76SF00515.

\section{REFERENCES}

${ }^{1}$ E. Marx, Deutches Reichspatent No. 455933

${ }^{2}$ A. Krasnykh, LINAC2000, p. 772 